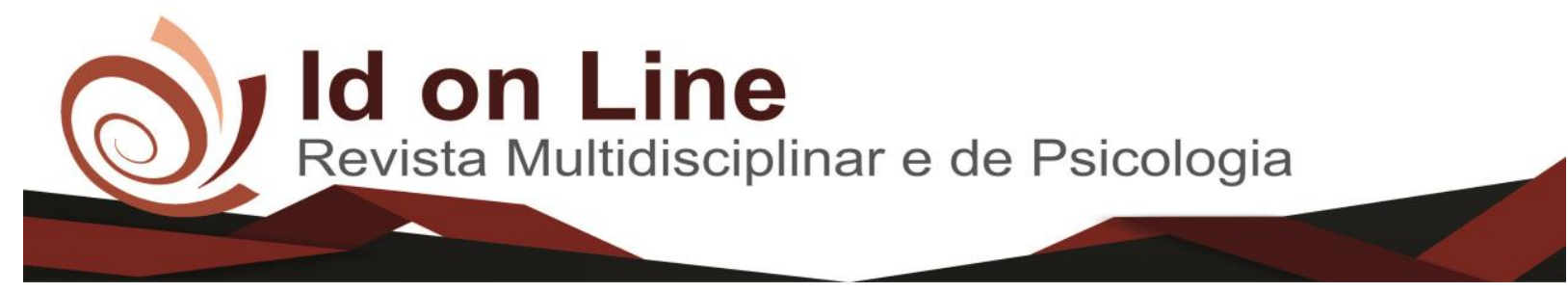

Artigo

\title{
Determinação do Teor de Captopril 25mg por Espectrofotometria de Absorção Molecular - UV, comercializado em Farmácias de Manipulação de Vitória da Conquista/BA
}

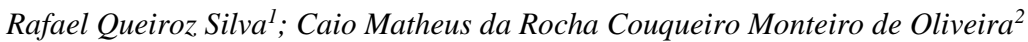

Resumo: Sendo indicado para o tratamento da hipertensão, insuficiência cardíaca, infarto do miocárdio e nefropatia diabética, o captopril é uma droga anti-hipertensiva e vasodilatadora que pode ser encontrada nas farmácias do país, na forma de cápsulas, de $12,5 \mathrm{mg}, 25 \mathrm{mg}$ ou $50 \mathrm{mg}$, doses que variam especificamente pela variação da quantidade do captopril existente em cada fórmula. Dentro dessa ótica, a finalidade do presente trabalho foi analisar o controle de qualidade, bem como ponderar a função do anti-hipertensivo em questão nas farmácias magistrais, com base na avaliação do perfil de doseamento do fármaco em questão. Metodologia: foi realizada análise do captopril $25 \mathrm{mg}$, que foram denominados de A, B e C, através da espectrofotometria de absorção molecular UV (ultravioleta). O fármaco em apreço foi obtido por meio de farmácias magistrais de Vitória da Conquista/BA. Resultado: feita a pesquisa do captopril 25mg, obtemos o teor de 92,76\% para o fármaco A, $112,98 \%$ para o B e $105,88 \%$ para o C, pelo que se conclui que o fármaco em questão não se encontra em padrões

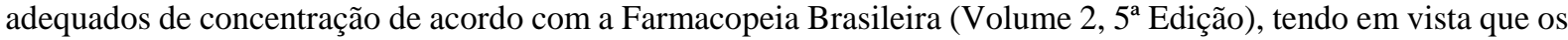
valores obtidos ficaram fora do mínimo/máximo permitidos, 97,5\% e 102,0\%, respectivamente, em relação à substância analisada.

Palavras-chaves: Captopril, controle, doseamento, magistral, qualidade, teor.

\section{Determination of the Captopril Content 25mg by Molecular Absorption Spectrophotometry - UV, sold in Manipulation Pharmacies of marketed in Pharmacies Vitória da Conquista in Bahia}

\begin{abstract}
It is indicated for the treatment of hypertension, heart failure, myocardial infarction and diabetic nephropathy, captopril is an antihypertensive and vasodilator drug that can be found in pharmacies in the country, in the form of capsules, $12.5 \mathrm{mg}, 25 \mathrm{mg}$ or $50 \mathrm{mg}$, doses that vary specifically by varying the amount of captopril in each formula. Within this perspective, the purpose of the present study was to analyze the quality control, as well as to consider the role of the antihypertensive in question in the master pharmacies, based on the evaluation of the dosing profile of the drug in question. Methodology: Captopril 25mg analysis was performed, which were denominated A, B and C, through UV (ultraviolet) molecular absorption spectrophotometry. The drug in question was obtained through magistrate pharmacies of Vitória da Conquista / BA. As a result, captopril 25mg was investigated, $92.76 \%$ for drug A, $112.9 \%$ for B and $105.7 \%$ for $\mathrm{C}$, and it is concluded that the drug in question is not present in appropriate standards of concentration according to the Brazilian Pharmacopoeia (Volume 2, 5th Edition), considering that the values obtained were outside the minimum / maximum allowed, 97.5\% and $102.0 \%$, respectively, in relation to the substance analyzed.
\end{abstract}

Key-words: Captopril, control, dosage, master, quality, content.

\footnotetext{
${ }^{1}$ Graduando de farmácia pela Faculdade Independente do Nordeste. Contato: raffaellqueiroz@ gmail.com

${ }^{2}$ Graduação em Ciências Farmacêuticas pela Universidade Estadual de Feira de Santana e mestrado em Quimica pela Universidade Estadual do Sudoeste da Bahia. Atualmente é professor da Faculdade Independente do Nordeste.
} 


\section{Introdução}

As formas farmacêuticas sólidas comumente utilizadas e conhecidas pela população, por meio da via oral, são adquiridas por meio do medicamento industrializado ou através das farmácias de manipulação, estas por meio de cápsulas produzidas especificamente para cada paciente, de acordo com a prescrição médica de cada indivíduo. (SANTOS, 2012).

Assim, o processo de terapêutico do fármaco dependerá, além das questões fisiológicas de cada paciente, da precisão da fórmula produzida através da farmácia magistral. Logo, a análise do teor do fármaco é uma ferramenta imprescindível para avaliação e estudo das propriedades biofarmacotécnicas do mesmo e consequente eficácia terapêutica ao fim a que se destina. (BERNARDES et al., 2010).

Inúmeros são os medicamentos produzidos nas farmácias de manipulação, que são buscados em sua maioria em virtude do custo benefício se comparado aos medicamentos industrializados, estando nesse rol o captopril em cápsulas, que tem função primordial de antihipertensivo e vasodilatador na insuficiência cardíaca congestiva, atua na minoração do infarto agudo do miocárdio, na nefropatiadiabética, dentre outras enfermidades. (CLETO, 2012; KATZUNG et al., 2014).

A técnica da espectrofotometria na região do ultravioleta é utilizada para definir a porcentagem do teor do captopril em cápsulas. Na Farmacopeia Brasileira $5^{\text {a }}$ Edição (F. Bras.) relata-se o comprimento de onda $212 \mathrm{~nm}$ e aplicação de ácido clorídrico 0,1 M como meio de dissolução. (Farmacopeia Brasileira, 2010).

A referida técnica, espectrofotometria na região do ultravioleta, é imprescindível, ainda, para precisar a porcentagem do teor do captopril, medindo, pois, a qualidade das cápsulas que são manipuladas nas farmácias magistrais. (AZEVEDO et al., 2008).

Destarte, é de acordo com o teor do captopril encontrado em cada substância, podendo ser mínimo ao máximo ao valor de referência, que a eficácia terapêutica variará. Para valores acima da referência, por exemplo, poderá ocorrer intoxicação do paciente, dentre outros. De outro modo, para valores de referência abaixo do indicado, teremos uma ineficiente eficácia terapêutica, o que fará com que a enfermidade que se pretende tratar não seja feita da forma adequada. (PEIXOTO et al., 2005).

Desse modo, o estudo em tela tem como propósito ponderar o controle de qualidade, verificando o teor do captopril manipulado nas farmácias magistrais da cidade de Vitória da 
Conquista/BA, tendo como base o padrão de referência escolhido, buscando proporcionar resultados satisfatórios quanto à qualidade dos fármacos. (PASA, 2008).

O método de controle de qualidade utilizado foi o da espectrofotometria de absorção ultravioleta (UV), com emprego de cápsulas de captopril $25 \mathrm{mg}$ adquiridos das farmácias magistrais denominadas de A, B e C, com uso dos dados de referência fornecidos pela Farmacopeia Brasileira (2010), que vão de 97,5\% a 112,9\% do valor declarado do captopril. (BRASIL, 2007).

\section{Base Conceitual}

Em relação à atuação do fármaco captopril e os inibidores da enzima conversora de angiotensina compreende-se que os inibidores da enzima conversora de angiotensina reduzem a pressão arterial principalmente ao diminuir a resistência vascular periférica. (SILVA et al., 2013).

O captopril, objeto de estudo, se originou do veneno da jararaca, substância essa que possuía característica principal de diminuir a tensão, daí porque é utilizado precipuamente para tratar a hipertensão arterial e a insuficiência cardíaca congênita, dentre outras doenças. (VALENTINI, 2002).

O fármaco em estudo, captopril, é equivalente "ao 1-[(2S)-3-Mercapto-2-metil-1oxopropil]-L-prolina (Figura 1), e tem na sua estrutura como grupo funcional mercaptana, amida e carboxila. Apresenta como características físico-químicas: partículas cristalinas de cor branca ou quase branca. Solubilidade em água, etanol, clorofórmio e metanol. Com ponto de fusão variando de $105^{\circ} \mathrm{C}$ a $108^{\circ} \mathrm{C}$ (BRASIL, 2010)”. (MARCATTO, 2005).

Sendo a via oral a mais comumente utilizada pelos brasileiros para a ingestão de medicamentos, o captopril se apresenta no mercado por meio de comprimidos e cápsulas, estas últimas produzidas nas farmácias de manipulação, que, assim como os comprimidos, encontram-se cápsulas de 12,5 mg, 25 mg e 50 mg. (BERNARDES et al., 2010)

As indicações clínicas do captopril são: I) A Hipertensão Arterial Sistêmica (HAS), patologia que se tipifica por altos níveis de pressão arterial (PA) no indivíduo; II) O Infarto Agudo do Miocárdio, que é causado através do entupimento de uma artéria coronária por um coágulo de sangue; III) A Nefropatia Diabética, doença que provoca a perda de proteínas por meio da urina, já que os vasos sanguíneos do rim estão afetados; IV) Insuficiência Cardíaca, 
provoca dificuldades ao coração em bombear sangue em quantidade necessária à manutenção saudável do corpo humano. (KATZUNG, et al., 2014).

Sendo uma droga de larga utilização pela população brasileira, especialmente em virtude da sua eficiência no tratamento das patologias que são indicadas (hipertensão, insuficiência cardíaca, infarto do miocárdio e nefropatia diabética), é de suma importância o estudo do controle de qualidade do captopril, objeto do presente trabalho. (MUELLER, 2010).

O controle de qualidade tem relevante papel nas inúmeras etapas de fabricação de um fármaco. É imprescindível avaliar adequadamente as matérias-primas que serão usadas, assim como os demais produtos utilizados no processo de fabricação. Tais fatores, somados ao controle apropriado dos processos de produção, são indispensáveis para a obtenção de um remédio que tenha a segurança e eficácia a que se destina. (LISBINSKI, 2008).

Até por esse motivo, o próprio termo denominado de controle de qualidade e utilizado para englobar os princípios e normas que precisam ser seguidos pelos fabricantes e autoridades, objetivando em primeiro lugar a qualidade dos fármacos. Assim, o objetivo primordial do controle de qualidade é alcançar medicamentos mais precisos, eficazes e seguros. (MENEGHIN, 2007).

\section{Metodologia}

Para determinação do teor de captopril das formulações magistrais foi utilizada a técnica de espectrofotometria de absorção molecular UV. O espectrofotômetro utilizado foi o (QUIMIS U2M) selecionado o comprimento de onda $212 \mathrm{~nm}$. A solução estoque foi preparada pesando-se $0,5 \mathrm{~g}$ do captopril em um béquer dissolvendo-se com $\mathrm{HCl}$ 0,1 mol/L e transferido para um balcão volumétrico de 0,5 L obtendo uma solução de 1000 mg/L (solução estoque).

A curva de calibração foi construída utilizando-se os padrões com as seguintes concentrações: $5,10,20,25$ e $30 \mathrm{mg} / \mathrm{L}$. No preparo dos padrões pipetou-se $0,5 \mathrm{~mL}$ (5 mg/L), 1,0 mL (10 mg/L), 2,0 mL (20 mg/L), 2,5 mL (25 mg/L) e 3,0 mL (30 mg/L), usando pipeta volumétrica e tendo o cuidado de completar o volume do balão volumétrico de 100,00 $\mathrm{ml}$ com ácido clorídrico $0,1 \mathrm{~mol} / \mathrm{L}$ usando pipeta de Pasteur.

Foram coletadas amostras em três farmácias da cidade sendo denominadas como farmácias A, B e C. Cada cápsula da amostra magistral de captopril foi aberta num béquer de $50 \mathrm{~mL}$, homogeneizando com ácido clorídrico 0,1 mol/L e depois transferiu-se todo o conteúdo 
para um balão volumétrico de 50,00 mL posteriormente feita filtração à vácuo para remoção dos excipientes e pipetado um volume de $10,00 \mathrm{~mL}$ e transferido para um novo balão de 50,00 $\mathrm{mL}$ para finalizar a diluição.

\section{Resultados e Discussão}

Como pode ser observado na figura 1 que a curva de calibração do captopril apresenta uma boa faixa de linearidade apresentando coeficiente de regressão linear $\mathrm{r}^{2}=0,998$, valor este o qual encontra-se dentro dos parâmetros exigidos (BRASIL, 2003).

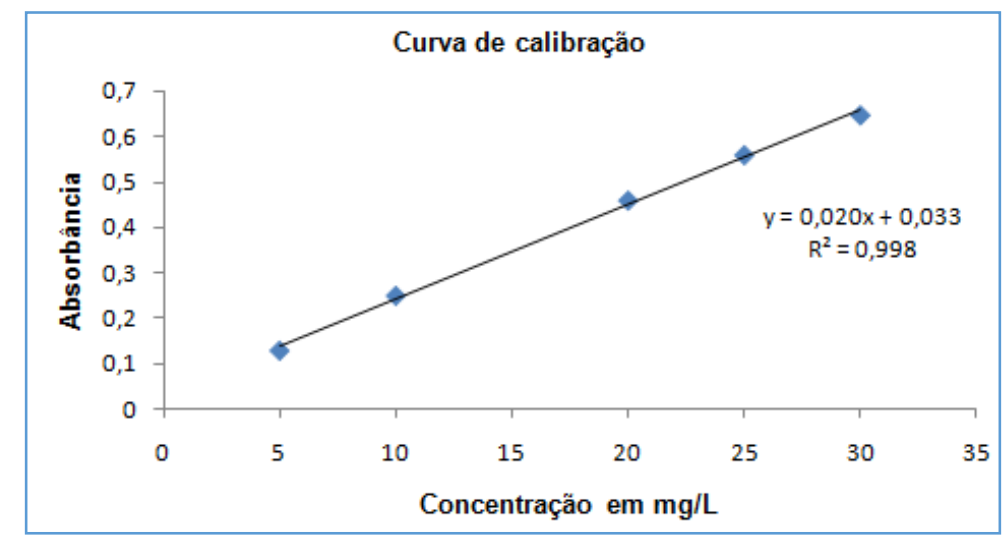

Figura 1: Curva de calibração do captopril.

Fonte: Dados da pesquisa.

Foram utilizadas 03 farmácias denominadas de A, B e C, sendo utilizadas 03 amostras para cada. Após a análise feita foi alcançado os seguintes resultados: Farmácia A - amostras com massas de 22,75; 22,75 e 24,08 mg respectivamente, no qual se encontrou um média do teor de pureza de $92,76 \%$, prosseguindo o processo de diluição com as farmácias B, alcançandose amostras de resultados 28,08; 28,50 e 28,16 mg, respectivamente, com média de teor de 112,98\%. Farmácia C obtendo-se amostras de resultados 26,67; 27,66 e 25,08 mg, com média de teor de $105,88 \%$.

Na pesquisa feita, o teor de captopril a ser encontrado nas cápsulas é entre 97,5 (mínimo) e 102,0\% (máximo). Após a análise de cada amostra estudada, observa-se que as amostras de captopril manipuladas nas farmácias de Vitória da Conquista/BA não estão de acordo com as especificações determinadas pela Farmacopeia Brasileira, 2010. 
Isso porque, a amostra A, que obteve teor de $92,77 \%$, está com o princípio ativo abaixo da concentração referida, não se prestando, pois, para alcançar a finalidade terapêutica a que se destina. De outro modo, as amostras B e C, que obtiveram teores de $112,9 \%$ e $105,88 \%$, respectivamente, estão com princípios ativo acima da concentração referida, podendo levar a intoxicação.

De acordo com a tabela 1 pode-se observar os dados referentes ao trabalho realizado.

Tabela 1 - Resultado de analise de amostras do captopril.

\begin{tabular}{ccccc}
\hline Amostra & $\begin{array}{c}\text { Massa de captopril } \\
(\mathrm{mg})\end{array}$ & $\begin{array}{c}\text { Teor }(\%) \\
\text { Intervalo de } \\
\text { aceitação }(\mathrm{mg})\end{array}$ & Resultado \\
\hline A & 23,19 & 92,77 & $97,5-102$ & Reprovado \\
B & 28,25 & 112,98 & $97,5-102$ & Reprovado \\
C & 26,47 & 105,88 & $97,5-102$ & Reprovado \\
\hline
\end{tabular}

Fonte: Dados da pesquisa.

Em virtude de tais fatos, por exemplo, é que se atribui um baixo consumo do captopril manipulado nas farmácias magistrais em estudo, uma vez não atingir o objetivo máximo que se espera, seja pela concentração abaixo ou acima do esperado.

Ainda assim, o estudo em questão, torna-se relevante para a avaliação do teor do medicamento, que, de acordo com a concentração encontrada, demonstrará se é seguro e se eficaz no tratamento para o qual é indicado. (GIL et al., 2007).

Logo, o teor do princípio ativo do fármaco não pode ser superior ou inferior aos limites admissíveis, pois, caso contrário, ocasionará problemas a saúde dos pacientes que o ingerirem. (CLETO, 2012).

Ademais, os testes de equivalência farmacêutica são estimulados em virtude dos fármacos estarem em desacordo com a sua fórmula original, tendo em vista que o partir dos valores encontrados (abaixo ou acima do valor de referência) é que chegaremos à conclusão de comprometimento, ou não, da bioequivalência e biodisponibilidade, o que afeta a qualidade garantida pelos laboratórios que o fabricam. (PUGENS et al., 2008)

Portanto, a qualidade dos medicamentos deve ser precisamente verificada, garantindo a segurança que deles se espera. Isso porque, o produto necessita estar de acordo com os padrões definidos pela Farmacopeia Brasileira. Caso isso não ocorra, o laboratório fabricante do fármaco poderá ficar com a sua credibilidade abalada perante o mercado de consumo e, pior, 
poderá sofrer risco iminente de perder o registro do produto e a sua licença de financiamento. (DOMICIANO et al., 2014).

\section{Conclusão}

O trabalho em apreço foi indispensável para avaliação do captoril $25 \mathrm{mg}$, determinando a sua qualidade e segurança, tendo em vista que os testes de qualidade desenvolvidos nas cápsulas das formulações A, B e C foram considerados satisfatórios em relação ao teor do captopril encontrados em cada amostra.

Assim, restou-se conclusivo que, como os valores obtidos de referência estavam abaixo (A) e acima (B e C) dos valores ideais indicados pela farmacopeia brasileira; os referidos medicamentos podem causar problemas à saúde dos pacientes, já que não possuem a eficácia esperada para os tratamentos que são indicados.

No caso dos valores de referência serem acima do valor ideal, a consequência será a intoxicação e outros riscos à saúde humana. Por outro lado, sendo os valores de referência abaixo do ideal, a principal consequência será a ineficácia terapêutica no controle da hipertensão arterial.

\section{Referências}

ANVISA (Brasil). Resolução RE no 899, de 29 de maio de 2003. Guia para validação de métodos analíticos e bioanalíticos. Diário oficial da República Federativa do Brasil, Poder executivo, Brasília, DF, 02 de julho de 2003.

MARCATTO, A.P.; Lamim, R.; Block, L.C.; Bresolin, T.M.B. Análise de cápsulas de captopril manipuladas em farmácias. Rev. Ciênc. Farm. Básica Apl., v. 26, n.3, p. 221-225, 2005, ISSN 1808-4532. Disponível em: <servbib.fcfar.unesp.br/seer/index.php/Cien_Farm/article/viewFile/427/410> Acesso em 09 setembro de 2017.

DOMICIANO, Samyra Leandro; FARIAS, Sandra Ramo; Análise de Qualidade de diferentes marcas de comprimidos de captopril. Revista Uniara. 17 (2). Disponível em: < http://www.revistarebram.com/index.php/revistauniara/article/view/31 > Acesso em 18 de outubro e 2017. 
MARTELETO, Beatriz Simões; VICENTINO, Rosimary Souza; GOMES, Sérgio Fernando de Oliveira. Análise de cápsulas de captopril provenientes de farmácias de manipulação do município de Barbacena. Rev. Med. Minas Gerais 2010; 20 (3 Supl 4): S31-S35 3. Disponível em < file:///C:/Users/Usuario/Downloads/v20n3s4a04\%20(1).pdf > Acesso em 11 setembro de 2017.

SILVA, Joel Rocha; VARGEM, Daiana da Silva; SIQUEIRA, Fernanda Silva; BORGES, Jéssica Alves. Avaliação da Concentração do Fármaco Captopril na Divisão de Comprimidos. V. 17, n.4, 2013, p. 9-16. Disponível em < file:///C:/Users/Usuario/Downloads/2310-8910-1-PB.pdf > Acesso em 08 setembro de 2017.

BERNARDES, Mariana Louise Peterson1; MUELLER, André2; GEBARA, Karimi Sater. Desenvolvimento e Controle de Qualidade de Cápsulas Contendo Captopril $25 \mathrm{Mg}$. $\begin{array}{llllllll}\text { Interbio } & \text { v.4 } & \text { n.1 } & 2010 \quad- & \text { ISSN } & \text { 1981-3775. Disponível em: }\end{array}$ <www.unigran.br/interbio/paginas/ed_anteriores/vol4_num1/arquivos/artigo2.pdf> Acesso em 28 de agosto de 2017.

AZEVEDO, Roberta de Cássia Pimentel; RIBEIRO, Gislaine Pereira, DE ARAÚJO, Magali Benjamin. Desenvolvimento e validação do ensaio de dissolução para captopril em cápsulas magistrais por CLAE. Revista Brasileira de Ciências Farmacêuticas BrazilianJournalofPharmaceuticalSciences, vol. 44, n. 2, abr./jun., 2008. Disponível em: <www.scielo.br/pdf/rbcf/v44n2/a11.pdf $>$ Acesso em 27 de agosto de 2017.

SANTOS, Diego Pereira Gabriel dos. Obtenção e caracterização de comprimidos de Captopril 50mg via compressão direta. [manuscrito] / Diego Pereira Gabriel dos Santos. 2012.28 f.: il. color. Acesso em 15 de agosto de 2017.

KATZUNG, B. G.; MASTERS, S. B., TREVOR, A. J. Farmacologia Básica e Clínica. 12. Ed. - Porto Alegre: AMGH, 2014.

VALENTINI, Sóstenes Rosa. Atributos da Validação da Metodologia Analítica do Captopril num Programa de Garantia de Qualidade. Dissertação de Mestrado. Florianópolis, 2002. Disponível em: https://repositorio.ufsc.br/bitstream/handle/123456789/82411/185613.pdf?sequence=1>

Acesso em 10 de agosto de 2017.

Agência Nacional de Vigilância Sanitária. Farmacopeia Brasileira. Volume 1, $5^{\text {a }}$ Edição, Brasília, 2010. Disponível em: < http://www.anvisa.gov.br/hotsite/cd_farmacopeia/pdf/volume\%201.pdf> Acesso em 02 de agosto de 2017.

CLETO, M. P. Avaliação da qualidade de comprimidos de captopril 50mg comercializados no Brasil. 2012. Araraquara, São Paulo/Sp. Acesso em 3 de outubro de 2017.

SKOOG, Douglas A; HOLLER, James F; NIEMAN, Timonthy. Princípios de análise instrumental. 5ed. Porto Alegre: Bookman, 2002. P. 115-137; 275-297. Acesso em 26 de agosto de 2017.

Bassi, A. B. M. S. "Conceitos fundamentais em espectroscopia". 
Vinadé, Maria Elisabeth do Canto; Vinadé, Elsa Regina do Canto, Métodos espectroscópicos de análise quantitativa. Editora UFSM.

HARRIS, C Daniel. Análise Química Quantitativa. Fundamentos da Espectrofotometria. 6ed. Rio de Jabeiro: LTC, 2005. P. 398-423.

PUGENS, Ana; DONADUZZI, Carmem; MELO, Eduardo; Controle de qualidade total de equivalência farmacêutica de três apresentações de captopril. Revista Eletrônica de Farmácia. Volume (1), 32-45, 2008.

GIL, E.S., ORLANDO, R.M., SERRANO, S.H.P., FISHER, D.C.H., MACHADO, S.A.S., MATIAS, R., BARA, M.T.F., SIRILO, H.N., FIGUEIREDO, G \& BARBOSA, W. G. Controle físico-química de qualidade de medicamentos. $2^{\mathrm{a}}$. Ed. São Paulo: Pharmabooks; 2007.

BRASIL. Agência Nacional de Vigilância Sanitária. RDC nº 67, de 08 de outubro de 2007. Dispõe sobre boas práticas de manipulação de preparações magistrais para uso humano em farmácias. Diário da República Federativa do Brasil, Brasília, 10 de out. 2007.

PASA, C. R. et al. Análise de medicamentos anti-hipertensivos contendo captopril, propranolol e losartana manipulados por farmácias de Campo Grande-MS. Revista Brasileira de Farmácia. 2008.

SOARES, A.K.A. et al. Biodisponibilidade comparativa de doses únicas de formulações de captopril. 2006. Disponível em: 〈http://www.unifor.br/notitia/file/759.pdf〉. Acesso em: 18 out. 2017

MOISÉS S. P. Tecnologia de produção de comprimidos. Disponível em: <http://. www.racine.com.br/download.asp?idarquivobanco=2122> . Acesso em: 18 de out. de 2017.

LINSBINSKI, L. M.; MUSIS, C. R,; MACHADO, S. R. P. Avaliação da equivalência farmacêutica de comprimidos de captopril. Revista Brasileira de Farmacia. 2008.

MENEGHIN, L. Z.; ADAMS, A. I. H. Avaliação físico-química de cápsulas de diazepam manipuladas em farmácias magistrais de Passo Fundo/RS. Revista Brasileira de Farmácia, 6770, 2007.

Como citar este artigo (Formato ABNT):

SILVA, Rafael Q.; OLIVEIRA, Caio M. da R. C. M. de. Determinação do Teor de Captopril 25mg por Espectrofotometria de Absorção Molecular - UV, comercializado em Farmácias de Manipulação de Vitória da Conquista/BA. Id on Line Revista Multidisciplinar e de Psicologia, 2017, vol.11, n.38, p.799-807. ISSN: 1981-1179.

Recebido: 08.11.2017

Aceito: 10.11 .2017 